II. Aus dem Stadtkrankenhause zu Dresden.

\title{
Das Verhalten des specifischen Gewichtes des Blutes bei Kranken.
}

Von Dr. Richard Schmaltz in Dresden.

Für die Bestimmung des specifischen Gewichtes des Blutes mit Benutzung kleinster Blutmengen stand bisher nur eine Methode zur Verfügung, die von Roy in Jahre 1884 beschrieben worden ist. Es wird dabei das zu untersuchende Blut in Probeflüssigkeiten von genau bekannter Dichtigkeit eingebracht und angenommen, dass in derjenigen Flüssigkeit, deren specifisches Gewicht gleich dem des Blutes ist, das letztere schwebell bleibt, ohne aufzusteigen oder abzusinken.

Roy verwendete als Probefüssigkeit Glycerinlösungen, andere haben später Gummilösung versucht u. s. w.

Gegen dieses Verfahren sind, wie mir scheint, zweierlei Einwendungen möglich. Einmal ist dasselbe ziemlich umständlich und zeitraubend. Man muss eine grosse Zahl von Lösungen in genau bekannter Dichtigkeit bereit halten und bei jeder Untersuchung mehrere Blutproben entnehmen, bis es gelungen ist, die entsprechende Probelösung herauszufinden; man muss ferver mit grosser Vorsicht zu Werke gehen, um zu vermeiden, dass beim Eimbringen des Blutes dieses letztere innerhalb der Lösung nach oben oder nach unten getrieben werde.

Zweitens aber scheint es mir zweifelhaft, ob überhaupt bei einer Flüssigkeit, die, wie das Blut, nicht eine Lösung, sondern eine Aufschwemmung specifisch schwererer corpusculärer Elemente in einem leichteren Medium ist, das specifische Gesammtgewicht in der beschriebenen Weise bestimmt werden kann.

Ich habe deshalb vor einem Jahre den Versuch gemacht, die pyknometrische Methode, die ja für Dichtigkeitsbestimmungen bei Flüssigkeiten die exacteste ist, zur Blutuntersuchung verwendbar zu machen, indem ich Capillaren von bestimmter Form und einem Rauminhalt von ca. 0,1 ccm als Pyknometer benutzte.

Bezüglich der Beschreibung dieses Verfahrens auf jene erste Veröffentlichung ${ }^{1}$ ) verweisend, will ich hier nur anführen, dass ich neuerlich Gelegenheit gehabt habe, die Exactheit der Methode, welche schon durch Controlluntersuchungen an einer Kochsalzlösung bewiesen worden war, bei einer Venaesection auf's neue zu prüfen:

Das specifische Gewicht des Aderlassblutes, welches einer Vene des linken Armes der Kranken entstammte, betrug, in einem grossen, 27,5 $\mathrm{g}$ fassenden Pyknometer bestimmt, 1,04098 (das Pyknometer war selbstverständlich mit blutwarmem Wasser geaicht worden); die capillarpyknometrische Bestimmung, an der rechten Hand und mit Benutzung einer anderen Waage vorgenommen, ergab 1,04148. Die Differenz war also nur 0,0005.

Dass die Dichtigkeit des Blutes durch Nahrungs- und Flüssigkeitsaufnahme (ja sogar durch die Zufuhr von 11 physiol. Kochsalzlösung), intensive Körperbewegung, Erzeugung von Hyperämie der Haut an der zur Blutgewinnung benutzten Stelle u. s. w. wenig oder garnicht beeinflusst wird, konnte schon früher mitgetheilt werden; hier sei es mir gestattet, nur noch die Ergebnisse von 55 Untersuchungen anzuführen, die ich im Laufe eines Jahres an mir selbst vorgenommen habe (Tabelle I), sowie die Resultate von Bestimmungen an 20 verschiedenen Personen beiderlei Geschlechts (Tabelle II).

\footnotetext{
1) Deutsches Arch. für klinische Medicin, B. 47. p. 145.
} 


\begin{tabular}{|c|c|c|c|c|c|c|c|c|c|c|c|c|c|}
\hline \multirow[b]{2}{*}{ D a t $\mathbf{u} \mathbf{m}$} & \multicolumn{13}{|c|}{ Tageszeite $\mathbf{n}$} \\
\hline & 7 & $\begin{array}{c}7^{1 / 2} \\
\text { Frühstück } \\
8 \mathrm{Uhr} \\
\end{array}$ & $8-9$ & $9-10$ & $11-12$ & $12-1$ & $\left|\begin{array}{c}1-2 \\
\text { Mittag- } \\
\text { essen 2 Uhr }\end{array}\right|$ & $2-3$ & $3-4$ & $4-5$ & $5-6$ & $6-7$ & $7-8$ \\
\hline 2. April 1890 & - & - & - & - & - & - & 1,0582 & 1,0603 & 1,0568 & 1,0583 & 1,0583 & 一 & 1,0570 \\
\hline 7. April 1890 & 一 & - & 1,061 & - & - & - & - & - & - & - & - & - & - \\
\hline 9. April 1890 & - & - & - & 1,059 & 1,059 & - & - & — & 1,061 & - & - & - & 1,059 \\
\hline 3. April 1890 & - & - & 一 & - & - & - & 1,0596 & 1,0596 & 1,0596 & - & - & 1,0596 & - \\
\hline 4. April 1890 & - & - & - & 一 & - & - & 1,0581 & 1,0578 & 1,059 & - & 一 & - & - \\
\hline 9. April 1890 & - & - & - & - & - & - & 1,0593 & 1,0584 & - & 1,0593 & - & - & - \\
\hline 30. April 1890 & $\rightarrow$ & - & - & - & - & 1,0588 & - & - & - & - & - & 一 & - \\
\hline 20. Mai 1890 & - & - & - & - & - & - & - & - & $\longrightarrow$ & 一 & 1,0593 & - & - \\
\hline 21. Mai 1890 & - & - & - & - & - & - & - & - & - & - & 1,056 & - & 1,059 \\
\hline 22. Mai 1890 & 1,0581 & - & - & - & - & 1,0597 & - & - & $\cdots$ & - & - & - & - \\
\hline 23. Mai 1890 & - & 一 & 一 & - & 1,0559 & - & - & - & - & - & - & - & - \\
\hline 24. $\mathrm{Mai} \quad 1890$ & - & 一 & - & - & - & - & - & - & - & 1,0572 & - & - & - \\
\hline 25. Mai 1890 & 1,060 & - & 1,0597 & 一 & 一 & - & - & - & - & - & - & - & 1,0593 \\
\hline 28. Mai 1890 & - & 一 & - & 一 & - & - & - & - & - & - & $\overline{-}$ & 1,0597 & - \\
\hline 31. Mai $\quad 1890$ & 一 & $\overline{-}$ & - & - & 一 & - & - & - & - & - & 1,0597 & - & - \\
\hline 1. Juni 1890 & - & 1,0621 & - & - & - & 1,0601 & - & - & - & - & - & - & 1,0589 \\
\hline 2. Juni 1890 & - & 1,0621 & - & - & - & - & 1,0589 & - & - & - & - & 1,0585 & - \\
\hline 9. Juni 1890 & - & - & - & 1,0605 & - & - & - & - & - & 一 & - & - & 1,0605 \\
\hline 10. Juni 1890 & 1,0613 & 1,0621 & - & - & - & - & - & - & - & - & - & - & - \\
\hline 9. März 1891 & 1,059 & - & - & - & - & - & 1,058 & - & - & 一 & - & - & 1,057 \\
\hline 10. März 1891 & 1,060 & - & - & - & 一 & - & 1,060 & - & - & - & - & - & 1,059 \\
\hline 13. Mărz 1891 & 1,061 & $一$ & 一 & - & - & - & - & - & - & 1,060 & - & - & $\longrightarrow$ \\
\hline 14. März 1891 & 1,059 & 一 & 一 & - & - & -- & 1,061 & 一 & - & - & - & - & 1,062 \\
\hline
\end{tabular}

Die durchschnittlich höheren Werthe in den Morgenstunden bestätigen eine frühere Mittheilung Reinecke's, der bei seinen Blutkörperchenzählungen an Gesunden in den Morgenstunden gleichfalls höhere Zahlen erhielt'1), und finden ein Analogon in Beobachtungen von Grosglik, welcher bei Hunden nach längerem Verweilen in gefesselter Lage ein Ansteigen der Blutconcentration fand $d^{2}$ ).

$\mathrm{T}$ a b e 11 e II.

\begin{tabular}{|c|c|c|c|}
\hline \multicolumn{2}{|c|}{$\mathrm{M}$ ä $\mathrm{n} \mathrm{n} \mathrm{e} \mathrm{r}$} & \multicolumn{2}{|c|}{ Frau $e n$} \\
\hline Alter & $\begin{array}{l}\text { Spec. Gew. } \\
\text { des Blutes. }\end{array}$ & Alter & $\begin{array}{c}\text { Spec. Gewicht } \\
\text { des Blutes }\end{array}$ \\
\hline $\begin{array}{lc}22 & \text { Jahr } \\
26 & \\
28 & \text { " } \\
32 & \text { " } \\
33 & \text { " } \\
35 & \text { " } \\
36 & \text { " } \\
56 & \text { " }\end{array}$ & $\begin{array}{l}1,058 \\
1,059 \\
1,058 \\
1,060 \\
1,059 \\
1,058 \\
1,059 \\
1,062\end{array}$ & 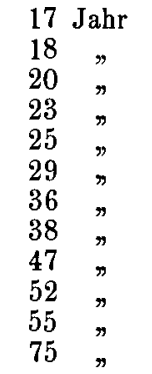 & $\begin{array}{l}1,057 \\
1,057 \\
1,057 \\
1,056 \\
1,055 \\
1,057 \\
1,055 \\
1,059 \\
1,057 \\
1,056 \\
1,055 \\
1,055\end{array}$ \\
\hline urchschnitt & 1,0591 & Durchschnitt & 1,0562 \\
\hline
\end{tabular}

Eine Bestätigung für die Constanz des specifischen Blutgewichtes bei Gesunden bieten auch die von Peiper kürzlich veröffentlichten, mit der capillarpyknometrischen Methode an 49 gesunden Erwachsenen und Kindern vorgenommenen Bestimmungen ${ }^{3}$ ). Um so auffallender ist es, dass Lloyd Jones, der gleichfalls an Gesunden arbeitete, so abweichende Resultate erzielte ${ }^{4}$ ), und ich möchte glauben, dass diese Differenz auf Rechnung der von Jones angewandten Methode (nach Roy, s. oben) zu setzen sei.

Durch die Güte meines früheren Chefs, des Herrn Geheimen Medicinalrath Dr. Fiedler, ist es mir nun ermöglicht worden, an einer grösseren Zahl von Kranken des hiesigen Stadtkrankenhauses Blutuntersuchungen anzustellen; es sei mir gestattet, ihm sowohl wie auch den Herren Hofrath Dr. Martini und Dr. Fischer, auf deren Abtheilungen ich gleichfalls einige Bestimmungen vornehmen durfte, an dieser Stelle meinen verbindlichsten Dank auszusprechen.

Als ich diese Untersuchungen unternahm, war mir, abgesehen von vereinzelten, in der Litteratur zerstreuten Angaben, auf dem in Rede stebenden Gebiet nur eine, im Mai des vorigen Jahres erschienene Abhandlung von Devoto bekannt, der in Prof. v. Jaksch's Klinik an einer Reihe von Kranken das specifische Gewicht des Blutes mit Hülfe der oben erwähnten Methode Roy's bestimmte ${ }^{5}$ ), aber unter 55 untersuchten Fällen nur bei 5 eine deutliche Herabsetzung

1) Virchow's Archiv Bd. 118.

2) Arch. de Physiol. normale et pathol. 5. S. II. 4. p. 705. 0ct. 1890.

$3)$ Ctrbl. für klin. Med. 1891 No. 12

4) Journ. of Physiol. VIII. Ref. in Schmidt's Jhrb. B. 215.

5) Zschr. für Heilkunde XI. 1890. derselben (unter 1,050) fand (offenbar infolge der Auswahl seines Materials). Ferner finden sich in einer mir später bekannt gewordenen Abhandlung von Prof. Quincke, über den Hämoglobingehalt des Blutes in Krankheiten, auch einige Angaben über das specifische Gewicht. ${ }^{1}$ )

Ich selbst liabe an 95 Kranken Bestimmungen der Dichtigkeit des Blutes vorgenommen und werde mir gestatten, über die gewonnenen Resultate in Kürze zu berichten.

Aeussere Verhältnisse brachten es mit sich, dass ich vorzugsweise weibliche Personen untersucht habe; theilweise geschah dies auch aus dem Grunde, weil das weibliche Geschlecht ja vorwiegend zur Erkrankung an acuten Anämieen neigt.

Tabelle III enthält die bei der Untersuchung chlorotischer, bezw. ohne bekannte Veranlassung an mehr weniger acuter Anämie erkrankter weiblicher Personen gewonnenen Resultate.

Tabelle III.

\begin{tabular}{|c|c|c|c|c|c|c|c|}
\hline No. & 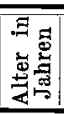 & Diagnos $\theta$ & $\begin{array}{l}\text { Speclf. } \\
\text { Gew. des } \\
\text { Bwutes }\end{array}$ & No. & 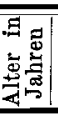 & Diagnose & $\begin{array}{l}\text { Spec } \\
\text { Gew. } \\
\text { Blnt }\end{array}$ \\
\hline 1. & 20 & & & & 20 & & \\
\hline 2 & 16 & & & & 18 & ria & \\
\hline 3. & 17 & lorosis & & & 17 & $\mathrm{Cr}$ & \\
\hline 4. & 24 & lorosis & & & 15 & $\mathrm{Cl}$ & 1 \\
\hline 5 . & 19 & sis & & & 16 & & \\
\hline & 19 & $s$ & & & 17 & gia & \\
\hline & 15 & & & & & & \\
\hline & 15 & loros & & & 22 & & \\
\hline 9. & 18 & lor & & & 20 & & \\
\hline & 21 & & & & & & \\
\hline & 25 & algia & & & 26 & & \\
\hline & 20 & s, Gastralgia & & & 19 & is, Gastralgia & 1,0 \\
\hline & 23 & & & 28 & 19 & is levis, Ga- & \\
\hline & 24 & & & & & & \\
\hline & & & 1,043 & & 15 & Chlorosis levis & 1,04 \\
\hline
\end{tabular}

Unter diesen 29 Kranken war bei 9 das specifische Blutgewicht unter 1,040 gesunken, und bei 2 sogar unter 1,035; die unter No. 1 angeführte Kranke ging wenige Wochen später geheilt und mit normalem Blutgewicht ab (Nickel s. unten).

Meine sehr knapp bemessene Zeit war die Ursache dafür, dass ich fast alle Kranken erst einige Tage und viele sogar erst 1 bis 2 Wochen nach ihrer Aufnahme in das Krankenhaus untersuchen konnte; ich bin aber überzeugt, dass bei der Untersuchung ausschliesslich frischer Fälle viel häufiger niedrige Zahlen gewonnen werden würden. Jedenfalls fanden sich bei den bisher mitgetheilten Fällen Werthe der Blutdichtigkeit, welche weit unterhalb der Norm liegen und noch beträchtlich niedriger sind, als die von Devoto veröffentlichten.

Nach Beendigung meiner Arbeiten gelangte nun noch das Referat eines von Hammerschlag in Wien über den in Rede stehenden Gegenstand gehaltenen Vortrags (Wiener med. Wochenschr. 1891 Nr. 1) und eine inzwischen erschienene Abhandlung von $\mathrm{Cope} \mathrm{mann}^{2}$ )

1) Virchow's Archiv B. 54 p. 537.1872.

2) Brit. med. Journ. 24. Jan. 1891 
zu meiner Kenntniss. Copemann benutzte, gleich Devoto, die von ihm modificirte Methode Roy's und fand bei Chlorosen (die Zahl der Fälle ist nicht angegeben) als niedrigste Zahl des specifischen Blutgewichts 1,041. Copemann bemerkt deshalb meiner Meinung nach mit Unrecht - , dass bei den oben erwähnten Beobachtungen Quincke's, der unter drei Chlorotischen einmal ein specifisches Gewicht von 1,035 fand, Fälle von perniciöser Anämie mit einbegriffen gewesen sein möchten.

Die schon erwähnte Mittheilung von Peiper hingegen, der 10 anämische und chlorotische Individuen untersuchte, enthält Resultate, die mit den meinigen vollkommen übereinstimmen: er constatirte bei 4 Kranken ein Blutgewicht unter 1,040, einmal 1,0329 und einmal 1,0303. Wahrscheinlich hätte auch Peiper noch mehr niedrige Werthe gefunden, wenn er nicht theilweise ein poliklinisches Material benutzt hätte. Aus Hammerschlag's Vortrag sind Einzelheiten über seine Befunde nicht ersichtlich.

Ich lasse nun (Tab. IV) einige Beobachtungen an Kranken folgen, welche durch bekannte Einflüsse anämisch geworden waren bezw. an schweren Erkrankungen des Blutes litten.

Tabelle IV.

\begin{tabular}{|c|c|c|c|c|}
\hline No. & Alter & Geschl. & D i a g nos e & $\begin{array}{l}\text { Spec. Gew. } \\
\text { des Blutes }\end{array}$ \\
\hline 30 & 26 & w. & Haematemesis . . . . . . . & 1,031 \\
\hline 31 & 22 & w. & $\# \quad . \quad \cdot \quad . \quad . \quad . \quad . \quad$. & 1,045 \\
\hline 32 & 36 & w. & Metrorrhagiae . . . . . . . . & 1,042 \\
\hline 33 & 15 & w. & Leukaemia. Endocarditis . . . . & 1,034 \\
\hline 34 & 42 & $\mathrm{~m}$. & Pseudoleukaemia lymphatica . . . & 1,053 \\
\hline 35 & 26 & w. & Septikaemia. Endocarditis . & 1,030 \\
\hline 36 & 62 & w. & Carc. ventr. et hepatis . . . . & 1,039 \\
\hline 37 & 43 & w. & Fibrosarcoma uteri. Ascites. . & 1,041 \\
\hline 38 & 35 & w. & Chyluria. . . . . . . . . & 1,045 \\
\hline 39 & - & w. & Nephritis acuti . & 1,048 \\
\hline 40 & 19 & w. & Nephritis chron. . . & 1,046 \\
\hline 41 & 30 & m. & 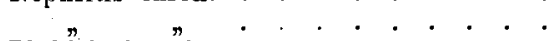 & 1,049 \\
\hline 42 & 22 & w. & Phthisis incipiens . & 1,036 \\
\hline 43 & 36 & m. & Phth. progressa. & 1,046 \\
\hline 44 & 33 & w. & $\begin{array}{llllllll} & 0 & & . & . & . & . & .\end{array}$ & 1,046 \\
\hline 45 & 33 & w. & Typh. abdom. (2. bis 3. Woche) . . & 1,044 \\
\hline 46 & 15 & w. & $\begin{array}{l}\text { med. } . . . \\
\text { (Recidiv, 7. Woche) 0titis }\end{array}$ & 1,046 \\
\hline 47 & 25 & w. & Insuff. valv. mitral. (Morb. Based ?) & 1,042 \\
\hline 48 & 17 & w. & Insuff. valv. mitral. . . . . . . & 1,044 \\
\hline 49 & 21 & w. & Insuff. valv. Aortae . . & 1,047 \\
\hline 50 & 20 & w. & Insuff. valv. mitral. . . . . . & 1,046 \\
\hline
\end{tabular}

Eine so geringe Dichtigkeit des Blutes, wie mehrere von den Chlorosen, zeigen unter den Fällen dieser Tabelle nur wenige. Darunter sind zwei Fälle von schwerer Erkrankung des Blutes (No. 33 und 35), welche beide innerhalb weniger Wochen nach der Blutuntersuchung gestorben sind, während die Chlorotischen nach relativ kurzer Zeit geheilt oder doch wesentlich gebessert entlassen werden konnten. Dagegen ist es auffallend, dass bei einer anderen Kranken (No. 32), die seit längerer Zeit an Metrorrhagieen schwerster Art litt, dadurch in hohem Grade anämisch geworden war und wenige Stunden vor der Blutuntersuchung einen bedenklichen Collaps nur mit Hülfe kräftiger Analeptica überstanden hatte, das Blutgewicht relativ hoch $(\mathbf{1}, 042)$ war. Sollten sich Beobachtungen dieser Art wiederholen, so würde darin vielleicht eine Stütze für die Auffassung der Chlorose als eigentliche Erkrankung des Blutes zu suchen sein, im Gegensatz zu den secundären, durch Blutungen entstandenen Anämieen (v. Hösslin hat bekanntlich neuerdings auf Grund der von ihm constatirten Anwesenheit von Hämatin und Eisen im Stuhlgang Chlorotischer, die Meinung ausgesprochen, dass die Bleichsucht eine Folge wiederholter Blutungen aus der Intestinalschleimhaut sei). ${ }^{1}$ ) In Fällen aber, wie No. 30, wo im Zusammenhang mit Blutverlusten eine excessive Erniedrigung der Dichtigkeit des Blutes beobachtet wird, würde man eine gleichzeitig bestehende, bezw. unter dem Einfluss der Blutungen entstandene Bluterkrankung anzunehmen haben.

Beträchtlich herabgesetzt ist das spec. Gewicht des Blutes ferner bei den beiden, durch maligne Tumoren kachectisch gewordenen Kranken; Peiper constatirte in einem entsprechenden Falle die ausserordentlich geringe Blutdichtigkeit von 1,0254 !

Dagegen hatten die beiden Typhuskranken, von denen die eine (No. 46) während des Recidivs eines mit eitriger Otitis media complicirten Falles in der 7. Krankheitswoche untersucht wurde, verhältnissmässig hohe Ziffern (1,044 bezw. 1,046); in Peiper's Fällen fand sich sogar ein mindestens normales Gewicht.

Die folgende Tabelle enthält nun noch eine Anzahl von Fällen verschiedener Art, bei denen das specifische Gewicht des Blutes keine oder nur eine geringe Herabsetzung, ja theilweise sogar eine abnorme Höhe zeigte.

l) Münch. med. Wochenschr. 1890. Ref. in Schmidt's Jahrb. 229

\begin{tabular}{|c|c|c|c|c|}
\hline No. & Alter & Geschl. & Diagnose & $\begin{array}{l}\text { Spec. Gew } \\
\text { des Blute }\end{array}$ \\
\hline 51 & 34 & w. & $\begin{array}{l}\text { Sehr anāmisches Aussehen (Alle Organe } \\
\text { gesund) }\end{array}$ & 1,058 \\
\hline 52 & 29 & w. & Dilatatio ventriculi. Anämia. . . & 1,052 \\
\hline & & w. & Ulcus ventriculi. Anämie. & 1,054 \\
\hline 54 & 64 & w. & Gastrectasie. Anämie. . & 1,057 \\
\hline 55 & 19 & w. & Ulcus ventriculi. & 1,056 \\
\hline 56 & 21 & w. & Jlcus ventriculi & 1,060 \\
\hline 57 & 57 & w. & Lues tert. stad. & 1,051 \\
\hline 58 & 30 & w. & ues tert. stad. & 1,056 \\
\hline 59 & 42 & m. & Phthisis progressa & 1,051 \\
\hline 60 & 20 & w. & Phthisis progressa . . . . . . . . & 1,055 \\
\hline 61 & 48 & w. & Phthisis pulm., Myoma uteri, Metrorrhagiae & 1,056 \\
\hline 62 & 13 & m. & Phthisis progressa . . . . . . & 1,062 \\
\hline 63 & 35 & w. & hthisis pulm. Gastrectasia & 1,053 \\
\hline 64 & 24 & w. & thisis progressa . . & 050 \\
\hline 65 & 19 & w. & Susp. Phthiseos. Anămia & 1,052 \\
\hline 66 & 16 & w. & Phthisis progressa . & 1,063 \\
\hline 67 & 16 & w. & Insuff. valv. mitral. Rheumat. art. & 1,060 \\
\hline 68 & 18 & w. & Insuff. valv. mitral. Rheumat. art. . & 1,051 \\
\hline 69 & 38 & w. & Insuff. valv. mitral. Cirrbos. hepat. Ascites & \\
\hline 70 & 29 & w. & Insuff. valv. mitral. Stenos. ost. venos. sin. & 1,060 \\
\hline 71 & 20 & w. & Insuff. valv. mitral. Stenos. ost. venos. sin. & 1,063 \\
\hline 72 & 26 & m. & Insuff. valv. mitral et valv. Aortae . & 1,058 \\
\hline 73 & 37 & & enos. ost. venos. sin. Dilatatio cordis & 1,057 \\
\hline 74 & 51 & & Insuff. valv. Aortae . & 1,056 \\
\hline 75 & 22 & & ost. pulmonalis . & 1,055 \\
\hline 76 & 73 & & us senilis . . . & \\
\hline 77 & 79 & & arasmus senilis . . . & 1,052 \\
\hline 78 & 79 & & Marasmus senilis. Lues & 1,052 \\
\hline 79 & 28 & w. & Combustio gravis (6. Tag) & 1,054 \\
\hline 80 & 48 & $\mathrm{~m}$. & Diabetes insipidus & 1,060 \\
\hline 81 & 15 & w. & Rheumatismus articulorum & 1,059 \\
\hline 82 & 20 & w. & Pleuritis exs. sin. (Reconval.) & 1,057 \\
\hline 83 & 15 & w. & Bronchitis acuta (Reconval.). & 1,059 \\
\hline
\end{tabular}

Der erste Fall dieser Tabelle betrifft eine Frau, die ein hochgradig anämisches Aussehen bot; ihre inneren Organe erschienen vollkommen normal (kein Eiweiss im Harn), und auch das Blut hatte nicht nur eine normale Dichtigkeit, sondern auch einen annähernd normalen Hämoglobingehalt $[\mathbf{7 0} \%$ der Fleischl'schen Scala $\left.\left.{ }^{1}\right)\right]$ und normale Blutkörperchenzahl (4 352 000). Das Gleiche gilt von den folgenden 3 Fällen, von denen besonders No. 52 und 54 sehr anämische und abgemagerte Personen betrafen; dennoch hatten auch diese Kranken ein normales Blutgewicht, und man darf daraus schliessen, dass auch der Hämoglobingehalt des Blutes wenigstens annähernd normal war (siehe unten). Nun hat kürzlich 0 ppenheimer ${ }^{2}$ ) an einer grösseren Zahl von Kranken nachgewiesen, dass nicht selten, trotz anämischen Aussehens, der Blutbefund, was Hämoglobingehalt und Anzahl der rothen Scheiben anlangt, normal gefunden wird, und nimmt zur Erklärung solcher Fälle eine abnorme Blutvertheilung in Anspruch. Die Hypothese 0 ppenheimer's, welche der Verfasser mit einer Reihe zutreffender Argumente gestützt hat, dürfte gewiss für viele Fälle zur Erklärung genügen (z. B. No. 30 unserer Tabelle); für andere Fälle ist doch aber, wie mir scheint, die Annahme einer wirklichen Oligämie sehr naheliegend und wohl auch zulässig. Dass im Zustande der Inanition die Blutmenge abnehmen könne, scheint mir aus einer Beobachtung von $\mathrm{Buntzen}$ [citirt bei $\left.\mathrm{Hosslin}{ }^{3}\right)$ ] hervorzugehen, der bei einem hungernden Hunde die Zahl der rothen Blutkörperchen ansteigen sah, eine Erscheinung, die doch wohl im Sinne einer Concentration des Blutes durch Verminderung seiner Gesammtmenge erklärt werden muss. Ganz analoge Ergebnisse hatten Fränkel und Röhmann bei hungernden Thieren ${ }^{4}$ ) und nehmen gleichfalls eine Eindickung des Blutes an, während andererseits Bizzozero und Torre bei fastenden Fröschen eine Vorminderung der Production rother Blutkörperchen fanden.5) Nun befand sich aber z. B. die Kranke No. 52 thatsächlich in einem chronischen Inanitionszustand; sie musste, wegen andauernder Magenbeschwerden mit häufigem Erbrechen, monatelang mit Magenausspülungen behandelt werden und wurde mit künstlichen Fleischpräparaten ernährt. No. 54 litt an einer enormen Magendilatation; auch sie war abgemagert und hatte eine anämische Haut. Ich möchte doch zweifeln, ob es richtig ist, in solchen und ähnlichen Fällen wegen des normalen Gehaltes des Blutes an Hämoglobin und Blutscheiben die Diagnose der Anämie auszuschliessen.

1) Nach den Untersuchungen von Mikulicz und Bierfreund ist der Durchschnitt bei Frauen $78 \%$ der Scala. Centralbl. für Chirurgie 1890 No. 25, Beilage.

2) Deutsche med. Wochenschr. 1889 No. 42-44.

3) Ueber den Einfluss ungenügender Ernährung auf die Beschaffenheit des Blutes. Münch. med. Wochenschr. 1890 No. 38-39.

4) Zeitschr. für physiol. Chemie IV. Ref. in Schmidt's Jahrb.

5) Virchow's Archiv, Bd. 95 
Das gleiche gilt, wie ich meine, wenn auch aus anderen Gründen, von den Phthisikern. Es ist höchst auffallend, dass gerade einige der schwersten Fälle von Lungenphthise ein normales, ja sogar ein relativ hohes Blutgewicht boten, während die Krauke No. 42 (Tabelle IV) im ersten Stadium der Phthise ihrem Blutbefund nach hochgradig anämisch war (specifisches Gewicht $=1,036$, Hämoglobingehalt $=35 \%$, Zahl der r. Blk. $=2972000$ ). Eine Inanition durch Nahrungsmangel könnte man für Phthisiker nur ausnahmsweise als Grund für diese Erscheinung annehmen, und auch die Consumption bietet dafür keine genügende Erklärung. Ich möchte vielmehr die Vermuthung aussprechen, dass es sich in diesen Fällen um Staungserscheinungen handelt.

Es ist schon von Oertel darauf hingewiesen worden ${ }^{1}$ ), dass die von Bamberger, Lichtheim und Thönniessen gefundene stärkere Concentration des Blutes Herzkranker theils auf einer vermehrten Transsudation, theils aber auch auf einer Ansammlung der corpusculären Elemente in der Peripherie beruhen möchte. Durch diese globulöse Stase ${ }^{\star}$, die neuerdings durch Experimente von Krüger eine Bestätigung erhalten hat (Krüger fand, dass bei der geringsten Stauung in einem Gefässgebiet der Gehalt des Blutes an festen Bestandtheilen zunimmt ${ }^{2}$ ), dürfte nun auch die Erscheinung zu erklären sein, dass bei Herzkranken bisweilen auffallend hohe Werthe des specifischen Blutgewichtes beobachtet werden, wie bei No. 70 und 71 unserer Tabelle (Krehl constatirte an dem Aderlassblute eines Kranken mit Stenose der Lungenarterie ein specifisches Gewicht von 1,071!:3). Eine Bestätigung findet diese Annahme in folgender Beobachtung: Fall 69, eine an Mitralinsufficienz und Lebercirrhose leidende Frau, hatte, ausserhalb des Bettes untersucht, ein Blutgewicht von 1,058, während dasselbe an einem anderen Tage, nach längerer Bettruhe, 1,054 betrug. Während also bei Gesunden unter dem Einfluss der Körperruhe das specifische Gewicht des Blutes eher ansteigt, batte es bei dieser Kranken beträchtlich abgenommen, offenbar infolge der durch die horizontale Lage bedingten Erleichterung des Abflusses in die Venen. Mit Rücksicht hierauf ist es aber ferner leicht denkbar, dass in ma nchen Fällen durch venöse Stauung ein normales Blutgewicht und ein lormaler Gehalt an Hämoglobin und an rothen Blutkörperchen vorgetäuschtwerde, während thatsächlich der Gelalt des Gesammtblutes an diesen Elementen herabgesetzt ist, dass also die betreffenden Kranken trotz ihres normalen Blutbefundes an ämisch sind.

In ähnlicher Weise, wie bei den Herzfehlern, kann derselbe Vorgang bei Phthisikern stattfinden, und es würde unter dieser Voraussetzung die an sich höchst quffallende Wahrnehmung erklärlich, dass gerade schwere Fälle mit ausgebreiteter Infiltration der Lunge bisweilen ein hohes Blutgewicht zeigen (vergl. No. 62 und 66).

lch möchte deshalb glauben, dass in allen den Fällen, welche mit einer irgend erheblichen Verlangsamung der peripheren Circulation einhergehen, die Resultate der Blutuntersuchung nur mit Reserve benutzt werden können, dass man zwar bei erheblicherHerabsetzung der Dichtigkeit u. s. w. einen anämischen Zustand annehmen, nicht aber bei normalem Befund denselben ohne weiteres ausschliessen darf.

$\mathrm{Ob}$ aus einer abnormen Erhöhung des specifischen Blutgewichts auf das Vorhandensein von Circulationsstörungen, bezw. bei Herzfehlern auf eine mangelhafte Compensation geschlossen werden darf, müssen weitere Beobachtungen lehren; jedenfalls würde dieser Schluss nahe liegen, wenn bei einem anämischen Herzkranken (wie No. 47, 48, 49, 50 in Tabelle IV) olne gleichzeitige Besserung des Gesammtbefindens das Blutgewicht anstiege.

Fragt man nun weiter, wodurch in den Fällen, welche eine theilweise so erhebliche Herabsetzung des specifischen Gewichtes des Blutes zeigen, diese letztere bedingt sein kann! so drängt sich als Antwort die Vermuthung auf, dass derselben eine Verminderung des specifisch schwersten Blutbestandtheils, der rothen Körperchen, zil Grunde liegen dürfte. In diesem Sinne sprechen sich auch Lloyd Jones ${ }^{4}$ und Hayem in seinem bekanten Werke über das Blut ${ }^{5}$ ) ans: "Je n'ai rien de particulier à dire sur la densité du sang, si ce n'est qu'elle dépend surtout de sa richesse globulaire."

In der folgenden Tabelle sind eine Anzahl Untersuchungen zusammengestellt, welche eine Vergleichung des specifischen Gewichts mit dem Gehalt des untersuchten Blutes an Hämoglobin und theilweise auch an rothen Körperchen gestatten. ${ }^{6}$ )

1) Therap. Monatsh. II, 6, 1888

Zeitschr. f. Biologie XXVI, 4, 1890

3) Deutsches Archiv ff klin. Med. XLIV p. 426

4) Brit. med. Journal 1888, II.

5 Dil sang. Paris 1889 , p. 400.

6 Der Hämoglobingehalt wurde nach v. Fleischl, in einigen Fällen nach $\mathrm{G}, \mathrm{w}$ ers abgeschätzt, die Blutkörperchen wurden nach T homa gezählt, mit A wzählung von 100 Feldern des Zeiss'schen Apparates.
Tabelle VI.

\begin{tabular}{r|c|c|c|c|c|c|c}
\hline \hline No. & $\begin{array}{c}\text { Spec. } \\
\text { Gew. }\end{array}$ & $\begin{array}{c}\text { Hämo- } \\
\text { globin } \%\end{array}$ & $\begin{array}{c}\text { Zahl der } \\
\text { rothen Blk. in } \\
\text { 1 cbmm }\end{array}$ & No. & $\begin{array}{c}\text { Spec. } \\
\text { Gew. }\end{array}$ & $\begin{array}{c}\text { Hämo- } \\
\text { globin } \%\end{array}$ & $\begin{array}{c}\text { Zahl der } \\
\text { rothen Blk. in } \\
\text { cbmm }\end{array}$ \\
\hline 1 & 1,035 & 30 & 3364000 & 27 & 1,044 & $35-40$ & 4068000 \\
2 & 1,036 & 35 & 2972000 & 28 & 1,044 & 60 & 4512000 \\
3 & 1,038 & 35 & 2728000 & 29 & 1,044 & 45 & 3520000 \\
4 & 1,038 & 35 & 4400000 & 30 & 1,045 & 45 & 3780000 \\
5 & 1,039 & 35 & 4144000 & 31 & 1,045 & $40-45$ & - \\
6 & 1,039 & $35-40$ & 2448000 & 32 & 1,045 & 50 & - \\
7 & 1,039 & 40 & 3352000 & 33 & 1,045 & $45-50$ & - \\
8 & 1,039 & 40 & - & 34 & 1,045 & $45-50$ & 3536000 \\
9 & 1,039 & 35 & 3360000 & 35 & 1,046 & $45-50$ & - \\
10 & 1,040 & $40-45$ & - & 36 & 1,047 & 50 & - \\
11 & 1,040 & $40-45$ & - & 37 & 1,047 & $40-45$ & - \\
12 & 1,041 & $35-40$ & 3380000 & 38 & 1,047 & $50-55$ & - \\
13 & 1,041 & $40-45$ & 2852000 & 39 & 1,048 & 55 & - \\
14 & 1,041 & $40-45$ & - & 40 & 1,049 & $45-50$ & 4164000 \\
15 & 1,041 & $30-35$ & 3604000 & 41 & 1,050 & 60 & - \\
16 & 1,042 & $40-45$ & 3440000 & 42 & 1,050 & $55-60$ & - \\
17 & 1,042 & 45 & - & 43 & 1,051 & 65 & - \\
18 & 1,042 & $35-40$ & - & 44 & 1,051 & 70 & 4464000 \\
19 & 1,043 & 50 & 3376000 & 45 & 1,052 & 65 & - \\
20 & 1,043 & $45-50$ & - & 46 & 1,053 & 75 & - \\
21 & 1,043 & 45 & - & 47 & 1,054 & $70-75$ & - \\
22 & 1,043 & 40 & - & 48 & 1,054 & $80-85$ & 4008000 \\
23 & 1,043 & 40 & 3876000 & 49 & 1,056 & 80 & 4664000 \\
24 & 1,044 & 50 & 4208000 & 50 & 1,056 & $70-75$ & 5176000 \\
25 & 1,044 & $45-50$ & 3096000 & 51 & 1,058 & 70 & 4352000 \\
26 & 1,044 & $40-45$ & - & & & & \\
& & & & & & & -
\end{tabular}

Aus dieser Tabelle ist ersichtlich, dass nach meinen Beobachtungen die Verminderung der Blutkörperchenzahl nur in so weit von Einfluss ist, als sie mit einer Herabsetzung des Hämoglobingehaltes einhergeht, dass aber, auch bei normaler oder nahezu normaler Menge der Blutscheiben das specifische Gewicht sehr niedrig sein kann, während in diesem Falle niemals ein hoher Hämoglobingehalt gefunden wurde (vergl. z. B. No. 3 und 4, 5 und 6, 24 und 25). Kurz bei den von niir untersuchten Kranken ging das specifische Gewicht des Blutes fast immer dem Gehalt desselben an Hämoglobin parallel, während es sich von der Anzahl der rothen Körperchen in weiten Grenzen unabhängig zeigte. Diese Wahrnehmung hat übrigens nichts auffälliges, wenn man bedenkt, dass ja das Hämoglobin vermöge seines Eisengehaltes die specifische Schwere der Blutscheiben bedingen muss.

Obgleich mir zur Prüfung des Hämoglobingehaltes nur ziemlich grobe Mittel zur Verfügung gestanden haben, und die gewonnenen Resultate der Nachprüfung bedürfen, so gewährt doch die ziemlich weitgehende Uebereinstimmung der einzelnen Untersuchungen einige Gewähr für deren Richtigkeit. Gestützt werden dieselben übrigens durch übereinstimmende Beobachtüngen von $\mathrm{Hammerschlag}$ (l. c. sowie durch eine Bemerkung Quincke's, die ich erst kürzlich bei der Durchsicht der erwähnten Abhandlung fand: ,Vergleicht man die gefundenen Hämoglobinprocente mit dem specifischen Gewichte, so ist ein Steigen und Fallen beider in gleichem Sinne unverkennbar ${ }^{*}$ Blutkörperchenzählungen sind weder von Hammerschlag noch von Quincke erwähnt (l. c.).

Beiläufig will ich bemerken, dass auch in meinen Fällen von Chlorose, wie dies schon früher von Gräber ${ }^{1}$ ) u. a. und neuerdings wieder von $\mathrm{Dowd}^{2}$ ) und Klein ${ }^{3}$ ) betont worden ist, in der Regel der Hämoglobingehalt relativ stärker herabgesetzt war, als die Blutkörperchenzahl.

Bei einem Theil der mir zur Verfügung gestellten, anämischen Kranken habe ich ferner das Verhalten des specifischen Gewichtes des Blutes bei der Heilung, bezw. Besserung der Anämie weiter verfolgt und, wie die folgenden Beispiele beweisen, gefunden, dass bei vier Fällen von, theilweise sehr hochgradiger Chlorose, die in ziemlich kurzer Zeit vollkommen geheilt wurden, die Dichtigkeit des Blutes von recht niedrigen Werthen rasch zunahm und endlich normal wurde. Bei mehreren anderen Kranken, welche vor vollendeter Heilung die Anstalt verliessen oder nach Beendigung meiner Beobachtungen noch in Behandlung blieben, war zwar ein mehr minder beträchtliches Ansteigen des Blutgewichtes zu constatiren, aber in keinem Falle erreichte dasselbe die normale Höhe, ohne dass auch die anderen Krankheitserscheinungen geschwunden wären. Ebensowenig ist bei einer im übrigen geheilten Kranken das specifische Gewicht des Blutes niedrig geblieben. (Vergl. die folgende Zusammenstellung.)

l) Therap. Monatshefte 1887, 10 .

) Americ. Journal of the medic. science. June 1890

Wiener med. Wschr. XL. 36-40, 1890 


\begin{tabular}{|c|c|c|c|c|}
\hline $\begin{array}{c}\text { Name } \\
\text { Alter } \\
\text { Diagnose }\end{array}$ & Datum & $\begin{array}{l}\text { Spec. } \\
\text { Gew. }\end{array}$ & $\begin{array}{c}\text { Hämoglobin } \\
0 \%\end{array}$ & $\begin{array}{l}\text { Zahl der } \\
\text { Blut- } \\
\text { körperchen }\end{array}$ \\
\hline $\begin{array}{l}\text { Nickel } \\
20 \text { Jahr } \\
\text { Chlorosis }\end{array}$ & 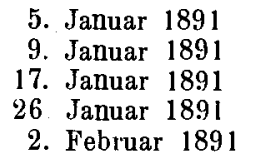 & $\begin{array}{l}1,030 \\
1,038 \\
1,043 \\
1,050 \\
1,053\end{array}$ & $\begin{array}{c}\overline{35} \\
45-50 \\
55-60 \\
75\end{array}$ & $\begin{array}{c}2 \overline{-} \\
- \\
- \\
-\end{array}$ \\
\hline $\begin{array}{l}\text { Kloust } \\
17 \text { Jahr } \\
\text { Chlorosis }\end{array}$ & $\begin{array}{l}\text { 10. December } 1890 \\
\text { 17. Januar } 1891 \\
\text { 28. Januar } 1891 \\
\text { 4. Februar } 1891\end{array}$ & $\begin{array}{l}1,044 \\
1,050 \\
1,051 \\
1,054\end{array}$ & $\begin{array}{c}45-50 \\
60 \\
65 \\
70-75\end{array}$ & $\begin{array}{c}3096000 \\
- \\
- \\
-\end{array}$ \\
\hline $\begin{array}{l}\text { Sauer } \\
16 \text { Jahr } \\
\text { Chlorosis }\end{array}$ & $\begin{array}{r}\text { 5. Januar } 1891 \\
\text { 19. Januar } 1891 \\
\text { 26. Januar } 1891 \\
\text { 2. Februar } 1891 \\
\text { 9. Februar } 1891 \\
\text { 16. Februar } 1891 \\
\text { 23. Februar } 1891\end{array}$ & $\begin{array}{l}1,033 \\
1,039 \\
1,039 \\
1,040 \\
1,042 \\
1,045 \\
1,046\end{array}$ & $\begin{array}{c}35-40 \\
35 \\
40-45 \\
40-45 \\
40-45 \\
45-50\end{array}$ & $\begin{array}{c}244 \overline{8} 000 \\
- \\
3440000 \\
-\end{array}$ \\
\hline $\begin{array}{l}\text { Seidel } \\
17 \mathrm{Jahr} \\
\text { Chlorosis }\end{array}$ & $\begin{aligned} \text { 28. } & \text { Januar } 1891 \\
\text { 4. } & \text { Februar } 1891 \\
\text { 11. } & \text { Februar } 1891\end{aligned}$ & $\begin{array}{l}1,035 \\
1,043 \\
1,044\end{array}$ & $\begin{array}{c}30 \\
40 \\
35-40\end{array}$ & $\begin{array}{c}3364000 \\
- \\
4 \times 68000\end{array}$ \\
\hline $\begin{array}{l}\text { Dōring } \\
20 \text { Jahr } \\
\text { Chlorosis } \\
\text { Gastralgia }\end{array}$ & 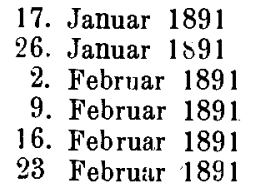 & $\begin{array}{l}1,{ }^{n} 41 \\
1,042 \\
1,042 \\
1,044 \\
1,047 \\
1,045\end{array}$ & $\begin{array}{c}30-35 \\
35-40 \\
40 \\
40 \\
40-45 \\
50\end{array}$ & $\begin{array}{c}3604000 \\
- \\
- \\
- \\
- \\
-\end{array}$ \\
\hline $\begin{array}{l}\text { Schmelz } \\
21 \text { Jahr } \\
\text { Chlorosis }\end{array}$ & $\begin{array}{l}\text { 11. December } 1890 \\
\text { 7. Januar } 1891\end{array}$ & $\begin{array}{l}1,041 \\
1,045\end{array}$ & $40-45$ & $\begin{array}{l}2852000 \\
3780000\end{array}$ \\
\hline $\begin{array}{l}\text { Turan } \\
24 \text { Jahr } \\
\text { Ohlorosis }\end{array}$ & $\begin{array}{l}\text { 10. December } 1890 \\
\text { 7. Januar } 1891 \\
\text { 19. Januar } 1891 \\
\text { 28. Januar } 1891 \\
\text { 4. Februar } 1891\end{array}$ & $\begin{array}{l}1,037 \\
1,046 \\
1,048 \\
1,048 \\
1,052\end{array}$ & $\begin{array}{l}- \\
- \\
55 \\
55 \\
65\end{array}$ & $\begin{array}{l}- \\
- \\
-\end{array}$ \\
\hline $\begin{array}{l}\text { Seifert } \\
24 \text { Jahr } \\
\text { Chlorosis }\end{array}$ & $\begin{array}{l}\text { 29. November } 1890 \\
\text { 15. December } 1890 \\
22 \text {. December } 1890\end{array}$ & $\begin{array}{l}1,043 \\
1,048 \\
1,051\end{array}$ & - & - \\
\hline $\begin{array}{l}\text { Eckoldt } \\
19 \text { Jahr } \\
\text { Chlorosis }\end{array}$ & $\begin{array}{l}\text { 12. Januar } 1891 \\
\text { 19. Januar } 1891 \\
\text { 26. Januar } 1891 \\
\text { 2. Februar } 1891 \\
\text { 9. Februar } 1891 \\
\text { 16. Februar } 1891 \\
\text { 23. Februar } 1891\end{array}$ & $\begin{array}{l}1,038 \\
1,039 \\
1,040 \\
1,041 \\
1,044 \\
1,044 \\
1,047\end{array}$ & $\begin{array}{c}35 \\
40 \\
40-45 \\
40-45 \\
40 \\
40-45 \\
50\end{array}$ & $\begin{array}{c}4400000 \\
- \\
- \\
- \\
- \\
-\end{array}$ \\
\hline $\begin{array}{c}\text { Gösel } \\
\text { 26 Jahr } \\
\text { Haematemesis }\end{array}$ & $\begin{array}{l}\text { 29. November } 1890 \\
\text { 15. December } 1890 \\
\text { 22. December } 1890 \\
\text { 29. December } 1890 \\
\text { 5. Januar } 1891 \\
\text { 12. Januar } 1891\end{array}$ & $\begin{array}{l}1,031 \\
1,035 \\
1,041 \\
1,041 \\
1,041 \\
1.044\end{array}$ & $\begin{array}{c}- \\
- \\
35-40 \\
- \\
-\end{array}$ & $\begin{array}{c}- \\
\overline{-} \\
3380000 \\
\overline{-}\end{array}$ \\
\hline $\begin{array}{c}\text { Scheffler } \\
18 \text { Jahr } \\
\text { Ch]orosis }\end{array}$ & $\begin{array}{l}\text { 14. Januar } 1891 \\
\text { 26. Januar } 1891 \\
\text { 4. Februar } 1891 \\
\text { 11. Februar } 1891 \\
\text { 19. Februar } 1891\end{array}$ & $\begin{array}{l}1,039 \\
1,038 \\
1,042 \\
1,043 \\
1,042\end{array}$ & $\begin{array}{c}35 \\
35 \\
45 \\
45 \\
40-45\end{array}$ & $\begin{array}{c}3360000 \\
- \\
- \\
-\end{array}$ \\
\hline $\begin{array}{l}\text { Kern } \\
25 \text { Jahr } \\
\text { Chlorosis }\end{array}$ & $\begin{array}{l}\text { 1. December } 1890 \\
\text { 29. Januar } 1891 \\
\text { 16. Februar } 1891\end{array}$ & $\begin{array}{l}1,041 \\
1,043 \\
1,047\end{array}$ & $\begin{array}{c}\overline{40} \\
50-5 \overline{5}\end{array}$ & $\begin{array}{c}- \\
3876000 \\
-\end{array}$ \\
\hline
\end{tabular}

Hämoglobingehalt des Blutes bestimmt, während es von der Anzahl der rothen Blutkörperchen in weiten Grenzen unabhängig ist. Bei der Besserung anämischer $\mathrm{Zu}$ stände steigt das specifische Gewicht des Blutes an und erreicht mit vollendeter Heilung die Norm; dasselbe scheint demnach als exacter Maassstab für die Beurtheilung des Krankheitszustandes verwendbar zu sein. Bei Verlangsamung der Circulation in den Extremitäten ist das specifische Gewicht des Blutes nicht selten erhöht und kann, trotz bestehender Anämie, normal gefundell werden (Herzfehler, Lungenleiden). specifische Gewicht des Blutes ziemlich genau dem Hämoglobingehalt parallel geht, und dass auch der letztere in zwei geheilten Fällen annähernd normal geworden ist (bei Turan fehlt an dem normalen Gewicht gleichfalls noch eine Kleinigkeit). Dem gegenüber möchte ich auf den Fall Schmelz hinweisen, in welchem die Anzahl der Blutscheiben in einer vierwöchentlichen Beobachtungszeit beträchtlich zugenommen hatte; der Hämoglobingehalt war aber nur wenig vermehrt, und dementsprechend auch das specifische Gewicht des Blutes nur um 0,004 angestiegen.

Ich bin mir der Lücken, welche die vorliegende Arbeit bietet, vollkommen bewusst und konnte selbstverständlich von vornherein nicht erwarten, zu einer umfassenden Bearbeitung des ganzen, noch wenig bekannten Gebietes mit meiner beschränkten Zahl von Beobachtungen imstande zu sein; vielleicht ist es mir aber gelungen, zu weiteren Untersuchungen auf demselben anzuregen.

Die bisher gewonnenen Resultate lassen sich in folgende Sätze zusammenfassen:

Während unter normalen Verhältnissen beim Menschen das specifische Gewicht des Blutes nur in engen Grenzen schwankt, ist dasselbe bei anämischen Zuständen in der Regelerheblich herabgesetzt, und zwar am meisten bei der Chlorose und den eigentlichen Erkrankungendes Blutes. Das Blutgewicht wird hauptsächlich durch den 